\title{
Natural Course of Early Radiological Signs of Femoroacetabular Impingement in An Asymptomatic Population
}

\author{
S Frontini, A Speranza, R Alonzo, S De Sanctis, Edoardo Monaco*, A Ferretti and C DArrigo \\ Università La Sapienza, II Facoltà di Medicina e Psicologia, Azienda Ospedaliera SantAndrea, Itlay
}

Submission:July 10, 2017; Published: July 18, 2017

*Corresponding author: Edoardo Monaco, Università La Sapienza, II Facoltà di Medicina e Psicologia, Azienda Ospedaliera Sant’Andrea, Rome, Itlay, Tel: 3933953711 69; Email: edoardomonaco76@gmail.com

\begin{abstract}
Background: During last years a lot of studies are investigating about femoroacetabular impingement (FAI) in athletes and its treatment. Few is known about the natural course of asymptomatic patients that present radiological FAI signs.

Purpose: The purpose of this study is to evaluate the radiological and clinical outcome of early-detected radiological signs predisposing towards FAI.

Study design: this is a cross sectional study.

Methods: All 2360 Computerized Tomography (CT) scans executed in our Emergency Department in 2006 were examined. Inclusion criteria were: age (20-40 years old), inclusion of both hips in the imaged range, absence of previous hip pathologies or trauma. The following bone abnormalities predisposing towards FAI were investigated: center edge angle, acetabular version angle, crossover sign. 44 patients (88 hips) were included in this study. At the 10-year follow-up, 42 patients were clinically evaluated using Hip Outcome Score. 19 patients also had repeated radiological examination.

Results: 34 out of 88 hips presented at least one radiological sign predisposing towards FAI. At follow-up, no patients developed symptoms. None of these 34 hips were found to have an increased number of signs at follow-up.

Conclusion: The role of early-detected radiological factors predisposing towards FAI remains unclear in asymptomatic patients and in the development time of FAI as a distinct clinical entity. The presence of radiographic signs of FAI is not predictive for the development of osteoarthritis at medium-long term.
\end{abstract}

Keywords: Femoroacetabular impingement; FAI; Young; Hip; Adult hip; Radiological signs

\section{Introduction}

Femoroacetabular impingement (FAI) is reported as a common cause of hip pain in young adults [1]. It acts as a precursor for early degenerative changes [2] and it is considered a major etiological factor in the development of osteoarthritis of the hip (OA) [3-5]. It is not thought as a disease per se, but rather as a pathomechanic process by which the human hip can fail [6]. FAI occurs as a result of the abutment of the femoral neck and the acetabular rim that causes cartilage damage, with anterior hip pain and progression to early osteoarthritis of the hip $[2,3,7]$. Ganz et al. [3] identified two mechanism of impingement, based on the pattern of chondral and labral lesions observed during surgical treatment, and they divided the types of lesions in three groups, which result in different patterns of articular damage $[1,2,8]$. The first one was related to changes of the acetabular rim with an overhanging of the anterolateral component that primarily produces a breakdown of the acetabular labrum and then articular damage. This type was named Pincer impingement, resulting to be more common in active middleaged women [1,3,9-11]. Pincer lesions may due to acetabular retroversion, where the antero lateral edge obstructs flexion, or coxa profunda, where the relative depth of the acetabulum is increased [2,7]. The second kind of impingement involves the femoral head that has a non-spherical appearance and comes in contact with the acetabular articular surface, or presents a reduction of the anterior neck offset with a consequent articular failure.

This type, named Cam impingement [2,7], is more common in physically active young males $[1,3,10,11]$. In this form there 


\section{Journal of Physical Fitness, Medicine \& Treatment in Sports}

is a relative preservation of the labrum. The third type, the most common, occurs from mixed Cam and Pincer pathology at the anterior femoral neck and anterior superior acetabular rim [2,7,11,12-17], although Cobb et al. [2] found that the acetabulum in the two forms of impingement were different from each other. Several studies showed that is common to see abnormal bony morphologies predisposing to FAI in contralateral asymptomatic hips in patients with unilateral FAI $[13,14]$, and that there is a prevalence of such risk factors for FAI in asymptomatic individuals [13]. However, in current literature there aren't studies focusing on the development of radiological risk factors for FAI into clinical evidence at medium-long term.

\section{Materials and Methods}

We investigated the prevalence of bony abnormalities predisposing to FAI, according to the measurement parameters established in current literature, and we evaluated the evolution of these FAI radiological risk factors and the associated appearance of clinical symptoms in young adults that have never referred any symptoms before. In order to avoid exposition to radiation we used data obtained from routine Computerized Tomography (CT) scans that include hip joints in the imaged range.In the course of this study, 2,360 pelvic CT scans were examined. These scans were sourced from emergency ward in-patients between January and December 2006. Only patients between 20 and 40 years old (at the date of the CT scans) were included. Exclusion criteria were: pelvic fractures, rheumatic diseases, established degenerative changes (i.e., joint-space narrowing, osteophytes, subchondral cystic, sclerotic changes) and evidence of any arthropathy that could cause secondary alteration of the hip joint.

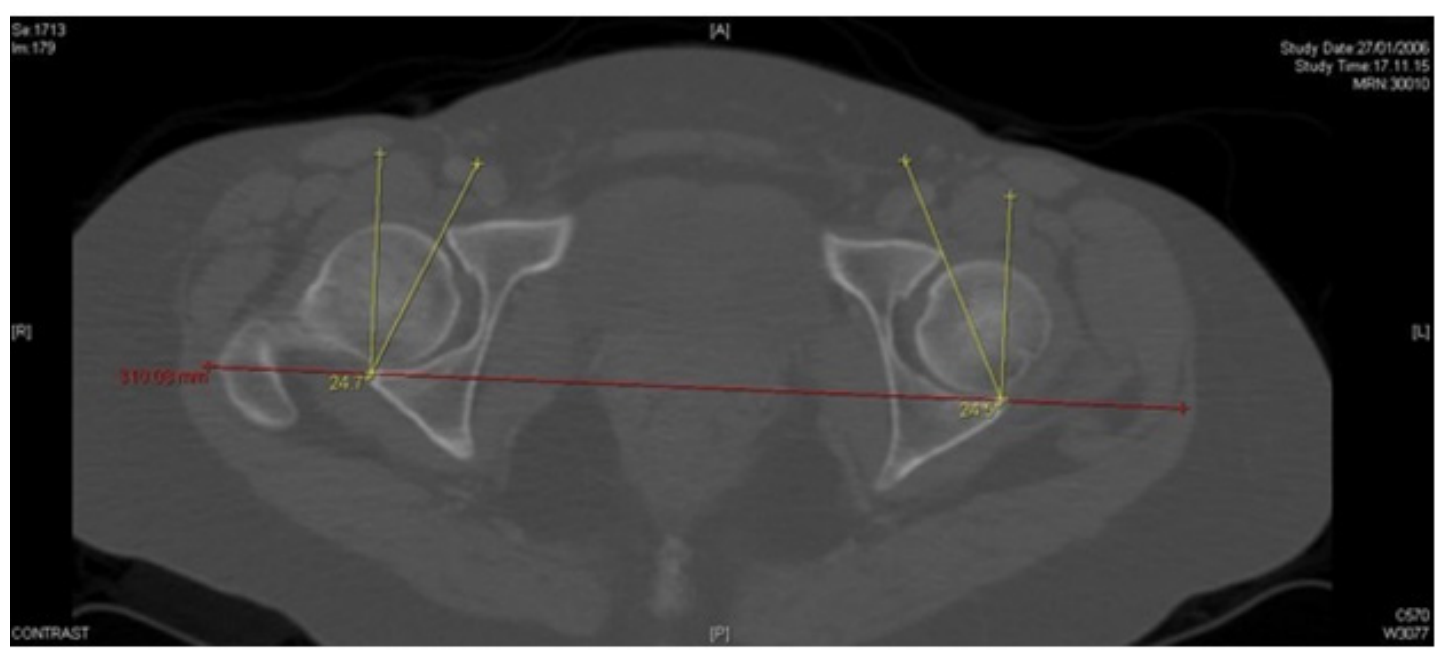

Figure 1: Acetabular version angle measurement.

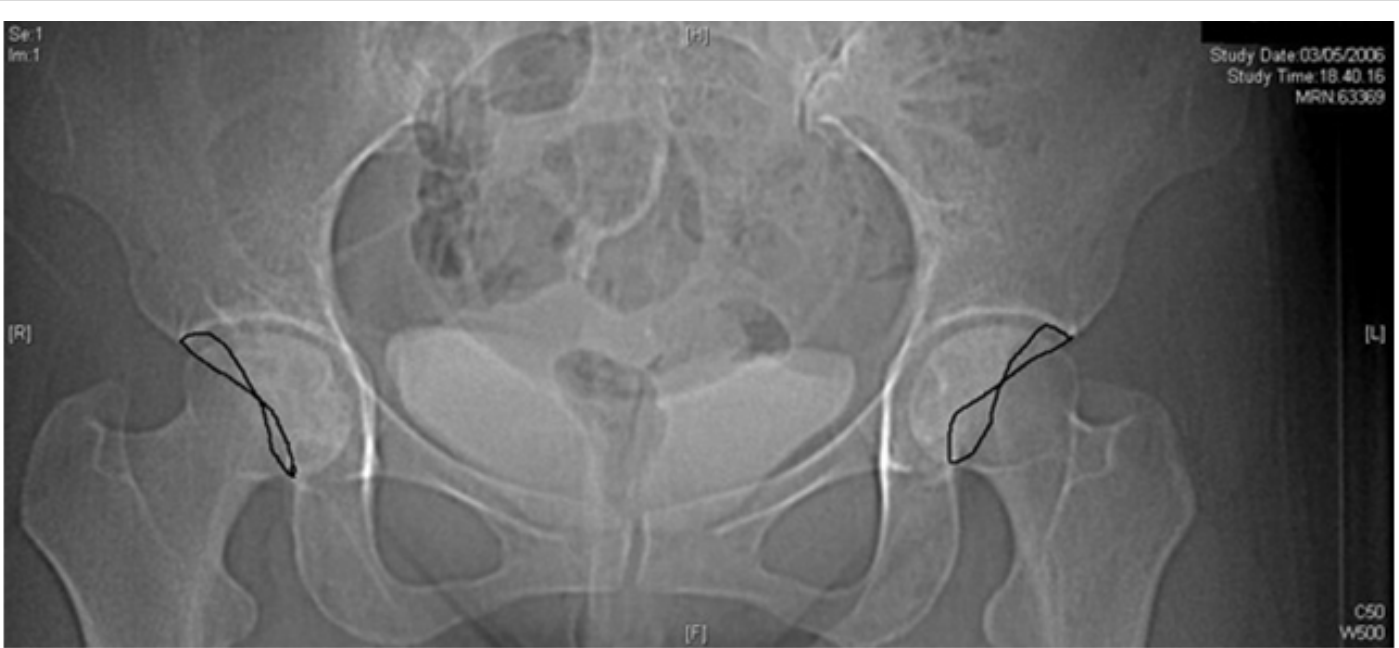

Figure 2: Presence of crossover sign in both hip joints, giving the "bow-tie" configuration. 
We obtained 44 relevant patients, 25 females and 19 males. On each one of the 88 hip joints we made the following measurements and observation: acetabular version angle, center edge angle, presence or absence of crossover sign. Version angle was evaluated on the orthogonal plane where the acetabulum cup is deeper, with two lines: the first one passing through the anterior and posterior rim of the acetabulum and the second one is perpendicular at the line passing through posterior acetabular rim bilaterally (Figure 1). The presence or absence of acetabular crossover sign was evaluated using the transparent 3-dimensional pelvis model, drawing the acetabular rim and checking if the anterior margin was crossing the posterior margin (Figure 2).
The center edge angle was evaluated on the same transparent 3-dimensional pelvis model (ensuring that the tip of the coccyx was midline and approximately $1 \mathrm{~cm}$ above the superior border of the pubic symphysis while making this observation) [18]: we evaluated the angle between the line passing through the femoral head center and the lateral rim of the acetabulum, and the line passing through both ischiatic tuberosities (Figure 3). Version angle values below $15^{\circ}$ define the condition of acetabular retroversion, center edge angle values over $40^{\circ}$ indicate the condition of coxa profunda (deep socket). Acetabular retroversion, coxa profunda, positive crossover sign were considered risk factors for FAI [12,13,19-23].

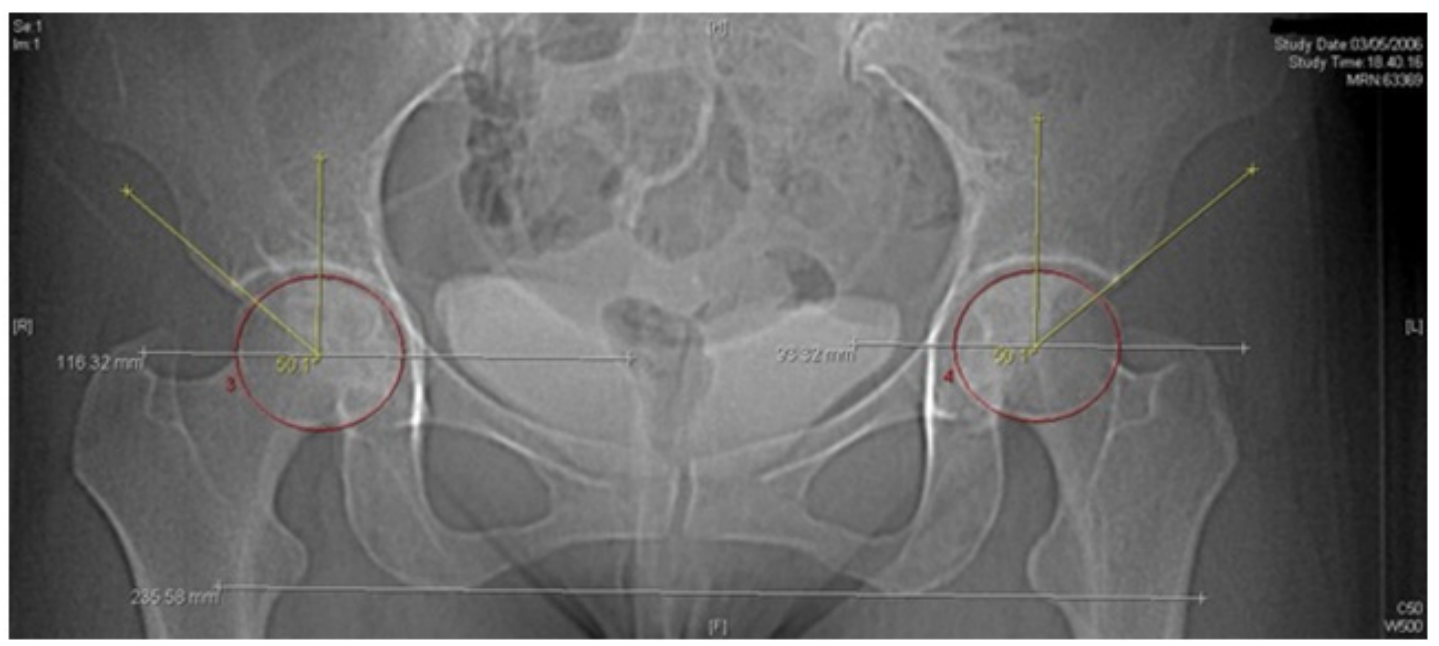

Figure 3:PresenceCenter Edge Angle: the angle between the line joining the lateral edge of acetabulum and the center of the femoral head and the vertical line that is perpendicular to the horizontal line joining the ischiatic tuberosities (white lines are parallel).

To find the incidence of the development of hip symptoms we administered clinical evaluation at a minimum follow up of 10 years (between December 2016 and January 2017). Forty-two patients were reviewed. Impingement tests (anteroposterior and posterorinferior), impingement sign test, Flexion Abduction External Rotation (FABER) provocation test were made $[1,3,4,7]$. In addition to the clinical evaluation, patients also completed the Hip Outcome Score (HOS) questionnaire as a measure of self-reported physical function [23]. We used two subscales for the evaluation: the activities-of-daily-living (ADL) subscale, containing items pertaining to basic daily activities, and the Sports subscale containing items pertaining to higherlevel activities, such as those required in athletics. On the ADL subscale, the item related to sitting and the item related to putting on socks and shoes were not scored. The response to each of the other 17 items were scored from 4 to 0 , with 4 being "no difficulty" and 0 being "unable to do". N/A responses were not counted. The score on each of the items was added together to get the item score total. The total number of items with a response was multiplied by 4 to get the highest potential score. If the subject answers all 17 items, the highest potential score is 68 . The item score total was divided by the highest potential score. This value was then multiplied by 100 to get a percentage.
The Sports subscale was scored in a similar manner with the highest potential score being 36 .

Moreover 19 patients at a minimum follow up of 10 years (between December 2016 and January 2017) had performed CT scans that included hip joints in the imaged range for diagnostic purposes not related to orthopedics. For each hip the same measurements previously achieved were made: center edge angle, presence or absence of crossover sign, acetabular version angle. We were able to make a comparison, which allowed us to investigate if the affected hip joints eventually increased the risk factors, and if there was a development of such risk factors in the hips that did not present radiological signs. In order to avoid any further radiation exposure no additional radiological examinations were performed. All the procedures were approved by the Ethical Commettee. All persons gave their informed consent prior to their inclusion in the study.

\section{Results}

The evaluation of 88 hips from 44 patients, 25 female and 19 male, demonstrated that 34 out of 88 joints (38\%), 15 of 50 (30\%) female joints (11 of 25 female participants), and 19 of $38(50 \%)$ male joints (13 of 19 male participants) had at least 
one morphologic aspect predisposing towards FAI among the following: acetabular version angle $<15^{\circ}$, presence of crossover sign, center edge angle $>40^{\circ}$. In 10 ( 6 males and 4 females) of 24 individuals (41\%) with at least one risk factor the findings were bilateral. Out of 88 joints, 7 presented more than one risk factor ( 4 out of 50 female joints and 3 of 38 male joints). Of those 7, 5 ( 3 female joints and 2 male joints) presented all the three risk factors together (Table 1). Two patients (1 male and 1 female) had all of the predisposing factors in both of their hip joints. Hence, according to the measurement parameters established in current literature, $68 \%$ of male and $44 \%$ of female asymptomatic patients in our study had at least 1 predisposing factor towards FAI in 1 or both of their hip joints.

Table 1: Results.

\begin{tabular}{|c|c|c|}
\hline 1 sign & 2sign & 3sign \\
\hline $\begin{array}{c}34(15 \mathrm{~F}, 19 \mathrm{M}) \\
\text { bilateral }\end{array}$ & $2(1 \mathrm{~F}, 1 \mathrm{M})$ & $5(3 \mathrm{~F}, 2 \mathrm{M}) 2$ bilateral \\
\hline
\end{tabular}

42 patients out of 44 ( 2 female patients died during the follow up) between December 2016 and January 2017 were reviewed and visited. No one referred typical FAI symptoms, such as groin pain associated with activity or insidious onset of pain in prolonged activities. Impingement tests were performed (anteroposterior, posteroinferior, FABER). For all the 42 patients the tests didn't elicit pain so the results were negative: the examination of the hip joints didn't reveal any limitation or decrease of range of motion, nor in the internal rotation and adduction in flexion nor in the extension and external rotation of the hip. In addition to the visit, the 42 patients completed HOS to assess a self-reported functional status. The subjects'self-reported ability to carry out ADL or practicing sports was normal in $100 \%$ of the participants of the study. Hence, the HOS score was $100 \%$ for all the patients for both ADL subscale and the Sports subscale.

We assumed that all the patients that underwent the visit received negative results in impingement tests at the clinical examination and none of them presented functional impairment of their hip joints. Moreover 19 out of 42 patients clinically evaluated, 11 that presented predisposing factors and 8 without risk factors, at a minimum follow up of 10 years (between December 2016 and January 2017) underwent CT scans that included hip joints in the imaged range. In none of the 38 hip joints (22 with radiological signs and 16 without radiological signs) there was evolution of the abnormal morphologies towards FAI or development of evident OA of the hip. None of the patients who presented radiographic characteristics in only one hip developed any contralateral evidence of predisposing factor during follow up. Also, none of them demonstrated an increase in the number of risk factors during follow up in the hip joints. Hence, the hips that presented one or two risk factors didn't develop a second or a third risk factor and the hips without radiological signs didn't develop evidence of risk factors at a medium-long term follow up of 10 years.

\section{Discussion}

In the recent years the understanding and awareness of FAI has become more widespread, making it one of the most investigated diseases in orthopedics. This is due to the possibility that the treatment of FAI may reduce progression to end-stage $\mathrm{OA}$ or delay the onset of hip symptoms [1,3,4,14-16]. Some studies focus on the conditions that can lead to morphologic changes predisposing the hip to FAI, such as coxa profunda, protrusio acetabuli, and acetabular retroversion [2,3,9,24], and how genetic factors can be responsible for these anatomic variations $[4,25,26]$. However, the presence of bony abnormalities in the asymptomatic hips suggests that additional variables, such as vulnerability of the labrum and articular cartilage to injury and physical activity level play an important role in this pathology $[13,14]$. The bony abnormalities responsible for FAI are of unknown prevalence, and many patients are asymptomatic and unaware of this subtle anatomical aberration [4]. Besides, in literature there is almost no information or clinical evidence on the development of such femoral and/or acetabular deformities predisposing towards FAI.

The first purpose of our study is to investigate the prevalence of bony abnormalities predisposing towards FAI in an asymptomatic population that underwent CT scans for nonorthopedic purposes, as the CT scan has a higher resolution than conventional radiographs to detect bony abnormalities [6]. Our study demonstrated that $30 \%$ of female joints and $50 \%$ of male joints had at least one morphologic aspect predisposing towards FAI using measurement parameters established in current literature. In $41 \%$ of the individuals with at least one risk factor, the findings were evident in both hips. Out of the 88 joints, $8 \%$ had more than one risk factor and $6 \%$ presented all 3 risk factors. The data obtained in this study confirm what is claimed in literature: there is a substantial prevalence of morphologic findings predisposing towards FAI in asymptomatic individuals, more frequent in male subjects than female, and the majority of the findings are evident in both hips [13].

The second objective of the study is to investigate the clinical and radiological evolution of such risk factors. As far as we could ascertain, this topic cannot be found in current literature. We followed up with the participants at a mid-long term check-up (after 10 years) to see what proportion of them eventually developed hip symptoms or evident FAI attributable to morphologic abnormalities. Most of the patients (42 of 44 included in the study) received a check-up and completed the HOS questionnaire. The Impingement tests were negative for all 42 patients. Also, no patients mentioned any limit in their ADL or sports attributable to the hip joint. The resulting HOS score was $100 \%$ for all subjects. At the same time we analyzed CT scans including hip joints in the imaged range that 19 patients had undergone at the mid-long term follow up (10 years). We were able to ascertain there has been no development of evident FAI in either the joints presenting risk factors or in 
the joints that didn't present risk factors. For each hip joint the same measurements previously made were checked as a comparison. The analysis stated that no increase of risk factors occurred and the hips without risk factors remain just as healthy. Although several studies in current literature evidence that the morphologic abnormalities we investigated can lead to impingement $[2,3,9,24]$, the results we obtained do not show the natural evolution towards FAI nor the development of clinical evidence over the mid-long term.

We acknowledge the weaknesses of this study. First, it only involved a small number of participants (44 patients). However, strict patients selection criteria allowed us to achieve an excellent randomization. Second, this was a retrospective study, with all the typical limitations of this type of study. The analysis was limited to hip imaging that was already required or completed in the past, as the additional radiation exposure required for this study could not be justified. Third, this study involved only a marginal investigation into extrinsic factors such as high athletic levels and demands. These factors are identified as potential sources for the development of clinical symptoms of FAI; however, even though all the patients selected are young adults, none of them stated that they are involved or have ever been involved in competitive sports activities.

On the other hand, the strengths of our study are the opportunistic use of raw data obtained from CT scans to assess hip joint morphologies characteristics, and the low dropout rate, with 42 subjects out of the 44 selected completing the study ( $5 \%$ loss in the mid-long term follow up). Moreover, as far as we can ascertain this is the only follow up study in literature that investigates the association between early radiological signs predisposing towards FAI and the development of clinical evidence.

\section{Conclusion}

On the basis of this study the significance of early radiological detected factors predisposing towards FAI remains unclear in asymptomatic patients, as well as time of development of FAI as a distinct clinical entity. Specific abnormalities associated with FAI are widely recognized as biomechanical risk factors for the development of OA of the hip, so a long term follow up in large numbers of patients may be useful to provide information on the natural course of FAI and the onset of hip symptoms.

\section{References}

1. Samora Julie Balch, Ng Y Vincent, Willis Thomas J (2011) Femoroacetabular impingement: a common cause of hip pain in young adults. Clin J Sport Med 21(1): 51-56.

2. Cobb Justin, Logishetty Kartik, Davda Kinner, Iranpour Farhard (2010) Cams and Pincer Impingement are distinct, not mixed. Clin Orthop Relat Res 468(8): 2143-2151.

3. Banerjee Purnajyoti, Mclean Christopher R (2011) Femoroacetabular impingement: a review of diagnosis and management. Curr Rev Musculoskeletal Med 4(1): 23-32.
4. Lavigne M, Parvizi J, Beck M, Siebenrock KA, Ganz R, et al. (2004) Anterior femoroacetabular impingement: part I. Techniques of joint preserving surgery. Clin Orthop Relat Res 418: 61-66.

5. Gekeler J (1978) Coxarthrosis with a deep acetabulum. Z Orthop Ihre Grenzgeb 116: 454-459.

6. Giori NJ, Trousdale RT (2003) Acetabular retroversion is associated with osteoarthritis of the hip. Clin Orthop Relat Res 417: 263-269.

7. Jaberi FM, Parvizi J (2007) Hip Pain in young adults: femoroacetabular impingement. J Arthroplasty 22(7 suppl 3): 37-42.

8. Parvizi Javad, Leunig Michael, Ganz Reinhold (2008) Femoroacetabular Impingement. J Am Acad Orthop Surg 15(9): 561-570.

9. Beck M, Kalhor M, Leunig M, Ganz R (2005) Hip morphology influences the pattern of damage to acetabular cartilage. J Bone Joint Surg $\mathrm{Br}$ 87(7): 1012-1018.

10. Ganz R, Parvizi J, Beck M, Leunig M, Nötzli H, Siebenrock KA (2008) Femoroacetabular impingement: a cause for osteoarthritis of the hip. Clin Orthop 417:112-120.

11. Pollard TCB, Villar RN, Norton MR, Fern ED, Williams MR, et al. (2010) Genetic influences in the aetiology of femoroacetabul impingement: a sibling study. J Bone Joint Sur Br 92(2): 209-216.

12. Pollard Thomas CB (2011) A perspective on femoroacetabular impingement. Skeletal Radiol 40(7): 815-818.

13. Kang Alan CL, Gooding Andrew J, Coates Mark H et al (2010) Computed Tomography assessment of hip joints in asymptomatic individuals in relation to femoroacetabular impingement. Am J Sports Med 38(6):1160-1165.

14. Samirul Imam (2011) Current concepts in the diagnosis and the management of femoroacetabular impingement. International Orthopedics 35(10): 1427-1435.

15. Leunig Michael, Beaulé Paul E, Ganz Reinhold (2009) The concept of femoroacetabular impingement. Clin Orthop Relat Res 467(3): 616622.

16. Byrd Thomas JW, Jones Kay S (2009) Arthroscopic femoroplasty in the management of Cam - Type femoroacetabular impingement. Clin Orthop Relat Res 467(3): 739-746.

17. Beck M, Leunig M, Parvizi J, Boutier V, Wiss D, et al. (2004) Anterior femoroacetabular impingement part II. Midterm results of surgical treatment. Clin Orthop Relat Res 418: 67-73.

18. Reynolds D, Lucas J, Klaue K (1999) Retroversion of the acetabulum: a cause of hip pain. J Bone Joint Surg Br 81(2): 281-288.

19. Ganz R, Leunig M, Leunig Ganz K, Harris WH (2008) The etiology of osteoarthritis of the hip: an integrated mechanical concept. Clin Orthop Relat Res 466(2): 264-272.

20. Tonnis D, Heinecke A (1999) Acetabular and femoral anteversion: relationship with osteoarthritis of the hip. J Bone Joint Surg Am 1999 81(12): 1747-1770.

21. Klaue K, Durnin CW, Ganz R (1991) The acetabular rim syndrome: a clinical presentation of dysplasia of the hip. J Bone Joint Surg 73(3): 423-429.

22. Macfarlane Robert J, Haddad Fares S (2010) The diagnosis and management of femoroacetabular impingement. Ann R Coll Surg Engl 92(5): 363-367.

23. Pollard Thomas CB, Villar Richard N, Norton Mark R, Fern ED, Williams MR, et al (2010) Femoroacetabular impingement and classification of the cam deformity: the reference interval in normal hips. Acta Orthopaedica 81(1): 134-141. 
24. Brian Pamela, Bernard Stephanie, Flemming Donald (2010) Femoroacetabular impingement: screening and definitive imaging. Semin Roentgenol 45(4): 228-237.

25. Antoniades L, Spector TD, MacGregor AJ (2001) The genetic contribution to hip joint morphometric and relationship to hip cartilage thickness. Osteoarthritis Cartilage 9(6): 593-595.
26. Martin RL, Kelly BT, Philippon MJ (2006) Evidence of validity for the Hip Outcome Score. Arthroscopy 22(12): 1304-1311.

\section{Your next submission with Juniper Publishers will reach you the below assets}

- Quality Editorial service

- Swift Peer Review

- Reprints availability

- E-prints Service

- Manuscript Podcast for convenient understanding

- Global attainment for your research

- Manuscript accessibility in different formats ( Pdf, E-pub, Full Text, Audio)

- Unceasing customer service

Track the below URL for one-step submission https://juniperpublishers.com/online-submission.php 